Background: Biologic therapies have revolutionised therapy in inflammatory diseases such as psoriatic arthritis (PsA), driving major improvements in outcomes. Th17 cells appear to play a key role in the pathogenesis of PsA, and IL-17 can trigger the release of chemoattractants such as CXCL8 and CCL20, leading to the further infiltration of other immune cells including neutrophils. Infiltrating activated neutrophils can themselves generate a range of chemoattractants which may amplify and sustain the inflammatory response. Therapeutic targeting of IL-17 with biologics such as secukinumab offers great benefit in PsA by blocking this inflammatory cycle: however the interaction of this agent with neutrophils, key components of host defence as well as potential mediators of this disease, is not known.

Objectives: This study aimed to measure key aspects of neutrophil function to determine: a) changes in the functions of circulating neutrophils in PsA patients pre-therapy, compared to age- and sex-matched healthy controls and $b$ ) if these functions changed in PsA patients 12-weeks post-secukinumab therapy.

Methods: Neutrophils were isolated from venous blood of 16 PsA patients and 10 healthy controls. Key neutrophil functions were measured at baseline and 12 weeks: reactive oxygen species (ROS) production, apoptosis (+/- TNF and GM-CSF), phagocytosis, receptor expression and chemotaxis. Changes in gene expression pre- and 12-weeks post-therapy ( $n=5$ PsA) were measured using RNAseq.

Results: PsARC response was observed in $70.6 \%$ of participants on secukinumab therapy at 12 weeks. There were no significant differences in ROS production, phagocytosis or chemotaxis in PsA patients at baseline (compared to healthy controls) or during therapy. Chemotaxis towards IL-8 in PSA patients at baseline was decreased compared to that of healthy controls, but this difference did not reach statistical significance. Surface levels of activation markers CD11b/CD18 and CD63 were increased in PsA patients at 12-weeks compared to baseline, while surface levels of CD16 decreased. RNA-seq analysis indicated down-regulation of pathways mediated by IL-17A, oncostatin M, TWEAK (TNFSF12) and CCL2 during therapy, but up-regulated expression of pathways involving de novo protein biosynthesis.

Conclusion: Therapy with secukimumab in PsA did not significantly affect neutrophil host defence functions. The changes that were seen in circulating neutrophils indicate selective up- and down-regulation of functions that may reflect potential alterations in local or systemic cytokines, and/or an increase in the circulating pool of activated neutrophils that are no longer recruited into sites of inflammation because of the down-regulation of the local IL-17/CXCL8 signalling network.

Disclosure of Interests: Andrew Cross: None declared, Jennifer Hawkes: None declared, Helen Frankland: None declared, Ayren Mediana: None declared, Helen Wright Grant/research support from: Novartis supporting this study, Nicola Goodson Grant/research support from: Novartis supporting this research, Steven Edwards Grant/research support from: Novartis supporting this work, Robert Moots Grant/research support from: Novartis supporting this work, Consultant of: a variety of companies including Novartis, Speakers bureau: a variety of companies including Novartis

DOI: 10.1136/annrheumdis-2020-eular.4477

\section{AB0116 DECREASED SERUM LEVEL OF IRISIN IN PATIENTS WITH ANKYLOSING SPONDYLITIS}

B. Nam ${ }^{1}$, S. Jo ${ }^{2}$, S. Lee ${ }^{3}$, T. H. Kim ${ }^{1,2} .{ }^{1}$ Hanyang University Hospital for Rheumatic Diseases, Department of Rheumatology, Seoul, Korea, Rep. of (South Korea); ${ }^{2}$ Hanyang University Institute for Rheumatology Research, Seoul, Korea, Rep. of (South Korea); ${ }^{3}$ Hanyang University Hospital for Rheumatic Diseases, Department of Radiology, Seoul, Korea, Rep. of (South Korea)

Background: Irisin, exercise-mediated myokine, is one of the most recently discovered hormones. Irisin has been shown to play multifunctional roles including anti-inflammation by suppressing secretion of NF kB, TNF-a, IL-6, and other pro-inflammatory cytokines from macrophages and adipocytes [1]. Thus, several attempts have been made to investigate irisin in chronic inflammatory rheumatic diseases. And recent evidences show that serum irisin concentration is lower in patients with osteoarthritis, rheumatoid arthritis, and behcet disease than health individuals [2-4]. Furthermore, one study showed that serum irisin level was negatively correlated with radiographic severity of knee osteoarhtiritis [2]. However, no previous study has investigated irisin in patients with ankylosing spondylitis (AS). Objectives: To assess the serum level of irisin, and evaluate the possible relationship of irisin with disease activity in patients with AS.

Methods: Male patients with AS fulfilled the modified New York criteria $(n=119)$, and healthy male controls $(n=30)$ were enrolled. Serum irisin level was measured by ELISA (Cusabio, CSB-EQ027943HU). Disease activity was assessed by acute phase reactants, Bath Ankylosing Spondylitis Disease Activity Index (BASDAI), Bath Ankylosing Spondylitis Functional Index (BASFI), and modified Stoke Ankylosing Spondylitis Spinal Score (mSASSS). Clinical characteristics and serum irisin level of the AS group were compared with those of the control group using Student t-test for normally distributed continuous measures and Mann-Whitney U test for non-normally distributed continuous measures. To evaluate the correlations of serum Irisin level and AS disease activity, Spearman's correlation test was used. AS patients were grouped into the high BASDAI group (BASDAI $\geq 4, n=45$ ) and the Low BASDAI group (BASDAI < 4, n=74). And serum irisin level was also compared between two groups.

Results: AS group had lower serum irisin concentration compared with healthy control group (60.50 [23.68-131.15] vs. 124.69 [79.58-192.90], $p=0.013)$, while age and body mass index were not significantly different between groups. There was no significant correlation between irisin level and disease activities. How ever, High BASDAI group showed significantly lower irisin level than low BASDA group (44.64 [18.13-85.89] vs. 65.68 [31.81-165.31], $\mathrm{p}=0.011$ ).

Conclusion: AS patients have lower serum irisin concentrations than healthy controls. AS patients with severe symptoms tend to have lower serum level of irisin than those with less severe symptoms.

\section{References:}

[1] H. Askari, et al. A glance at the therapeutic potential of irisin against diseases involving inflammation, oxidative stress, and apoptosis: an introductory review. Pharmacol Res. 2018

[2] Mao $\mathrm{Y}$, et al. Association of Irisin and CRP Levels with the Radiographic Severity of Knee Osteoarthritis. Genet Test Mol Biomarkers. 2016

[3] Rania M. Gamal, et al. Preliminary study of the association of serum irisin levels with poor sleep quality in rheumatoid arthritis patients. Sleep Med. 2020

[4] A. Icli, et al. Novel myokine: irisin may be an independent predictor for subclinic atherosclerosis in Behcet's disease. J. Investig. Med. 2016

Disclosure of Interests: None declared

DOI: 10.1136/annrheumdis-2020-eular.5034

\section{$\mathrm{AB} 0117$ \\ HLA B27`SUBTYPES FREQUENCIES IN COLOMBIAN PATIENTS WITH SPONDYLOARTHRITIS AND HEALTHY SUBJECTS TYPED IN HIGH-RESOLUTION TECHNIQUE}

C. Romero-Sánchez ${ }^{1,2}$, W. Bautista-Molano ${ }^{1,3}$, Y. M. Chamorro-Melo², A. Beltrán-Ostos ${ }^{4}$, J. De Avila ${ }^{1}$, J. M. Bello-Gualtero², A. Ramos-Casallas ${ }^{1}$, L. Chila ${ }^{1}$, C. F. Pacheco Tena ${ }^{5}$, P. Chalem ${ }^{6}$, C. Florez ${ }^{7}$, V. Parra-Izquierdo ${ }^{7}$, D. Jaimes ${ }^{4} .{ }^{1}$ Universidad El Bosque, School of Dentistry, Cellular and Immunology Group -INMUBO-, Bogotá, Colombia; ${ }^{2}$ Hospital Militar Central, Rheumatology and Immunology Department, School of Medicine, Bogotá, Colombia; ${ }^{3}$ Universidad Militar Nueva Granada, Clinical Immunology Group, Bogotá, Colombia; ${ }^{4}$ Clínicos IPS, Bogotá, Colombia; ${ }^{5}$ Investigación y Biomedicina de Chihuahua, Chihuahua, Mexico; ${ }^{6}$ Fundacion Instituto de Reumatología Fernando Chalem, Universidad El Rosario, Bogotá, Colombia; ${ }^{7}$ Gastroadvanced SAS, Bogotá, Colombia

Background: HLA-B ${ }^{\star} 27$ has been identify as a susceptibility and prognostic factor associated to axial spondyloarthritis. HLA-B ${ }^{\star} 27$ allele has been described to be present in about $90 \%$ of patients with ankylosing spondylitis, and with a different frequency in patients with other subtypes of SpA. In contrast, this allele has been observed to be present only in $7-8 \%$ in general population. A remarkable heterogeneity in $\mathrm{HLA}^{\star} \mathrm{B}^{\star} 27$ alleles has been reported. They have been deter mined at DNA sequence and some subtypes have been associated increasing the risk to develop the disease

Objectives: To establish the frequencies of HLA-B27 subtypes in a group of Colombian patients with SpA and healthy population

Methods: In total, 61 Blood samples from Colombian mestizo individuals with $\mathrm{SpA}$ according to ASAS classification-criteria were evaluated by Sequencing Technology: Illumina Sequencing/PacBio Sequencing with analysis of the second and third exon. Results reported with six digits (including null alleles). In total, 294 results of peripheral blood from healthy individuals without joint symptoms were analyzed. Frequencies were obtained for demographic and genetic variables. Ethic Committee approval code 2018-020/2017-023

Results: The SpA group had a mean age of $45,88 \pm 11,67,62.3 \%$ of them were male, $6.6 \%$ reported current smoking and $37.7 \%$ reported smoking sometime in life. In total, $67.2 \%$ had inflammatory back pain, $14.8 \%$ had dactylitis, $63.9 \%$ enthesitis and $57.4 \%$ arthritis. Thirty patients were HLA-B ${ }^{\star} 27$ positive with a genotypic frequency of $50.8 \%$ and an allelic frequency of $24.6 \%$. In this group of patients, the mean age was $43,5 \pm 11,8,76.6 \%$ were male, $86.7 \%$ of them were subtype $B^{\star} 27: 05: 02 \mathrm{~g}$ and $13.3 \%$ presented the B27:02:01g. None of the SpA patients had both $B^{\star} 27$ alleles.

On the other hand, the healthy individuals were men in $51.0 \%$ and the mean age was $37 \pm 15.4$ years. Ten subjects were positive for the HLA-B ${ }^{\star} 27$ allele with a genotypic frequency of $3.4 \%$ and an allelic frequency of $1.7 \%$. In this group of individuals $50.0 \%$ were male gender with a mean age of $38.4 \pm 17.9$ No individuals were found to have the two alleles or homozygous for the $B^{\star} 27$ allele. In all of them the subtype $B^{\star} 27: 05: 02 g$ was observed in high-resolution sequencing 
Conclusion: The SpA group had a mean age of $45,88 \pm 11,67,62.3 \%$ of them were male, $6.6 \%$ reported current smoking and $37.7 \%$ reported smoking sometime in life. In total, $67.2 \%$ had inflammatory back pain, $14.8 \%$ had dactylitis, $63.9 \%$ enthesitis and $57.4 \%$ arthritis. Thirty patients were $\mathrm{HLA}-\mathrm{B}^{\star} 27$ positive with a genotypic frequency of $50.8 \%$ and an allelic frequency of $24.6 \%$. In this group of patients, the mean age was $43,5 \pm 11,8,76.6 \%$ were male, $86.7 \%$ of them were subtype $B^{\star} 27: 05: 02 \mathrm{~g}$ and $13.3 \%$ presented the $B 27: 02: 01 \mathrm{~g}$. None of the SpA patients had both $B^{*} 27$ alleles.

On the other hand, the healthy individuals were men in $51.0 \%$ and the mean age was $37 \pm 15.4$ years. Ten subjects were positive for the HLA-B*27 allele with a genotypic frequency of $3.4 \%$ and an allelic frequency of $1.7 \%$. In this group of individuals $50.0 \%$ were male gender with a mean age of $38.4 \pm 17.9$. No individuals were found to have the two alleles or homozygous for the $B^{\star} 27$ allele. In all of them the subtype $B^{\star} 27: 05: 02 \mathrm{~g}$ was observed in high-resolution sequencing Acknowledgments: Hospital Militar Central (Grant 2017-023/2018-020), the Government Institute of Science, Technology, and Innovation, Francisco Jose de Caldas-COLCIENCIAS (Grant No. 130877757442) and Colombian Rheumatology Association (Grant-Conv-2019)

Disclosure of Interests: None declared

DOI: 10.1136/annrheumdis-2020-eular.3401

\section{AB0118 $\quad$ FREQUENCY OF INTESTINAL PARASITES AND THEIR ASSOCIATION WITH CLINICAL DISEASE ACTIVITY AND TREATMENT-DECISION IN PATIENTS WITH SPONDYLOARTHRITIS}

J. Chaparro-Olaya ${ }^{1}$, J. De Avila², W. Bautista-Molano ${ }^{2,3}$, L. Morales ${ }^{1}$, P. Hernandez ${ }^{1}$, A. Beltrán-Ostos ${ }^{4}$, M. León-Falla ${ }^{5}$, J. M. Bello-Gualtero ${ }^{6}$, A. Ramos-Casallas ${ }^{2}$, D. Acero-M ${ }^{3}$, C. Florez ${ }^{2,7}$, C. F. Pacheco Tena ${ }^{8}$, V. ParraIzquierdo ${ }^{2,7}$, Y. M. Chamorro-Melo ${ }^{6}$, C. Romero-Sánchez ${ }^{2,3,6} .{ }^{1}$ Universidad EI Bosque, Molecular Parasitology Laboratory, Bogotá, Colombia: ${ }^{2}$ Universidad El Bosque, School of Dentistry, Cellular and Immunology Group -INMUBO-, Bogotá, Colombia; ${ }^{3}$ Clinical Immunology Group, Hospital Militar Central, School of Medicine, Universidad Militar Nueva Granada, Bogotá, Colombia; ${ }^{4}$ Hospital Militar, Subdireccion de docencia e Investigación, Bogotá, Colombia; ${ }^{5}$ Universidad El Bosque, Master in Basic Biomedical Science Program, Bogotá, Colombia; ${ }^{6}$ Hospital Militar Central, Rheumatology and Immunology Department, Universidad Militar Nueva Granada, Bogotá, Colombia; ${ }^{7}$ Gastroadvanced SAS, Bogotá, Colombia; ${ }^{8}$ Investigación y Biomedicina de Chihuahua, Chihuahua, Colombia

Background: Studies of human intestinal microbiota have focused mainly on bacteria and scarce information on how eukaryotic parasites fit in the gut context or its role in human health and disease.

Objectives: This is an approach to explore if intestinal parasites represent a significant factor concerning the treatment-decisions or disease activity in inflammatory conditions such as SpA

Methods: A Cross-sectional study including 65 patients with $\mathrm{SpA}$ according to ASAS classification criteria was performed. Clinical evaluation was made by rheumatologists and gastroenterologists. Stool samples were collected and microscopically analyzed by direct saline, Mini Parasep concentration and Kato Katz. Most prevalent protozoa in Colombia were also analyzed using PCR/qPCR. Lab tests included fecal calprotectin, CRP, ESR, and HLA-B*27. The association between intestinal parasite infection and clinical/treatment variables were evaluated using the Chi-square or Fisher's exact test. (Ethical/ Cod.2017-023)

Results: SpA patients had a mean age of $43.9 \pm 11.5$ years, $61.5 \%$ were male, $52.5 \%$ were positive for HLA-B ${ }^{\star} 27$ and $87.7 \%$ had axial involvement. In total, $67.7 \%$ of the patients were receiving biological treatment, $64.6 \%$ had ASDAS-CRP $\geq 2.1$. In total, $75.4 \%$ of patients were positive for $\geq 2$ gastrointestinal symptoms with a predominance of abdominal pain $(66.2 \%)$, abdominal inflammation $(63.1 \%)$, diarrhea $(47.7 \%)$ and intolerance to some food $(58.5 \%)$. Interestingly, $21.3 \%$ have high levels of calprotectin, $20 \%$ of patients with high calprotectin were receiving biological treatment against IL-17 $(p=0.086)$ and $80 \%$ of these patients had BASDAI $>4(p=0.017)$ and ASDAS-VSG $>2.1 \quad(p=0.03)$.

The parasites found in SpA patients were Endolimax nana (98\%), Blastocystis ssp. (63.8\%), Entamoeba coli (8.6\%), Entamoeba histolytica (6.9\%), Chilomastix mesnili (6.4\%), E. dispar/moshkovskii (1.7\%) and Giardia intestinalis $(3.7 \%)$. Patients positive for $E$ coli $(80 \%)$ were treated with NSAIDs $(\mathrm{p}=0.003) .3 / 4$ of patients positive for $E$ histolytica presented HLAB*27:05:02 positive. Likewise, the only patient who was positive for $G$ intestinalis expressed this allele. $5 / 7$ of patients treated with Sulfasalazine presented Blastocystis ssp and $33.3 \% E$ coli. The presence of intestinal parasites in $\mathrm{SpA}$ patients was not associated with gastrointestinal symptoms, either disease-activity measures.

Conclusion: The intestinal parasitism in the tropical countries as Colombia have shown an interesting pattern in SpA patients. The treatment may modulate the presence of some parasites; however, the presence of intestinal parasites in SpA does not seem to influence clinical disease activity

Acknowledgments: The Government Institute of Science, Technology,and Innovation,Francisco Jose de Caldas-COLCIENCIAS(Grant No. 130877757442). Universidad El Bosque (PCl-2018-10091), Hospital Militar Central (Grant 2017023), Clínicos IPS, Gastroadvanced, Fundación Instituto de Reumatología Fernando Chalem-Bogota,Colombia and Biomedicina de Chihuahua, México

Disclosure of Interests: None declared

DOI: 10.1136/annrheumdis-2020-eular.3882

\begin{tabular}{|l|l|}
\hline AB0119 & SERUM LEVELS OF INTERLEUKIN-22 ARE HIGH \\
IN ANKYLOSING SPONDYLITIS, PARTICULARLY \\
IN SMOKERS, BUT DO NOT CORRELATE WITH \\
RADIOGRAPHIC BONE FORMATION NOR WITH \\
DISEASE ACTIVITY
\end{tabular}

M. Sagiv ${ }^{1}$, G. Slobodin ${ }^{2}$, T. Khatib ${ }^{2}$, I. Rosner ${ }^{2}$, M. Rozenbaum ${ }^{2}$, R. Peri ${ }^{2}$, F. Sabbah ${ }^{3}$, M. Adawi ${ }^{3}$, A. Kessel' ${ }^{2}{ }^{1}$ Azrieli Faculty of Medicine, Bar-llan University, Safed, Israel; ${ }^{2}$ Bnai Zion Medical Center, Haifa, Israel; ${ }^{3}$ Puria Medical Center, Tiberias, Israel

Background: Elevated serum levels of interleukin (IL)-22 were reported in patients with ankylosing spondylitis (AS) ${ }^{[1]}$ IL-22 was also reported to drive the osteogenic differentiation of mesenchymal stem cells. ${ }^{[2]}$

Objectives: To confirm the fact that serum levels of IL-22 are elevated in AS patients and to examine the relationship between concentrations of IL-22 and degree of radiographic progression in AS patients.

Methods: Seventeen male patients with established AS of more than 4 years duration signed the informed consent and donated $10 \mathrm{ml}$ of peripheral blood. Demographic data was collected from patient's charts. Disease activity indices were calculated for all patients and radiographic disease progression was calculated as mSASS. A control group included 6 healthy persons and 4 patients with advanced diffuse idiopathic skeletal hyperostosis (DISH). Serum levels of IL-22 were tested using enzyme-linked immunosorbent assay. Intergroup differences were examined using the Mann-Whitney test, while correlations were calculated using Pearson correlation coefficient.

Results: Serum IL-22 levels were remarkably elevated in patients with AS comparing to healthy individuals and patients with DISH $(p=0.005)$. However increased concentrations of IL-22 did not correlate with the degree of radiographic progression or AS disease activity indices, nor with disease duration or patient's age. Presence of diarrhea, psoriasis, uveitis, or elevated levels of $\mathrm{C}$-reactive protein did not influence the levels of IL-22 as well. More AS patients with elevated serum IL-22 were smokers $(p=0.05)$

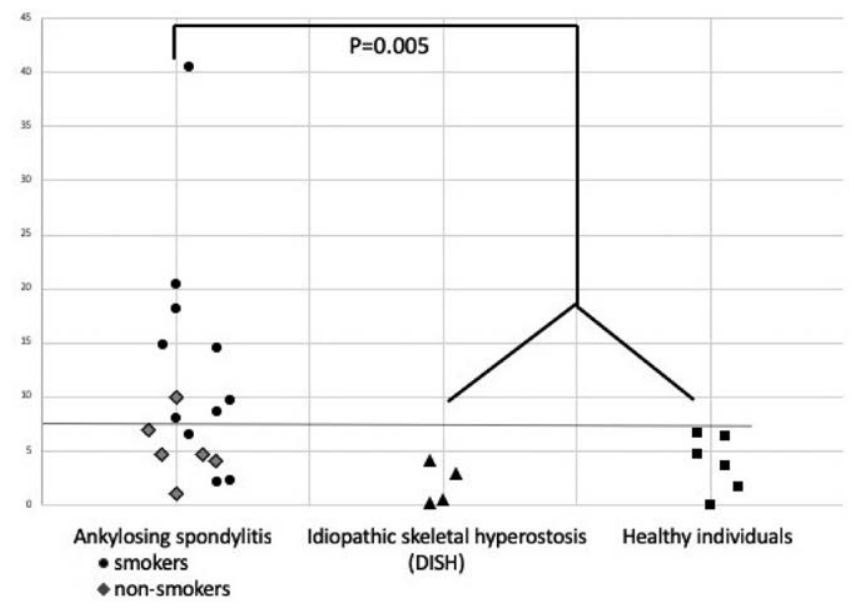

Fig. 1. Serum levels of interleukin $22(\mathrm{pg} / \mathrm{ml})$

Conclusion: The serum levels of IL-22 are elevated in patients with AS. It seems that smoking can be related to the elevated levels of serum IL-22 in AS. The significance of this data is unclear and further research is needed.

\section{References:}

[1] Zhang L, Li Y gang, Li Y hua, Qi L, Liu X guang, Yuan C zhong, et al. Increased frequencies of th22 cells as well as th17 cells in the peripheral blood of patients with ankylosing spondylitis and rheumatoid arthritis. PLoS One. 2012;7(4)

[2] El-Zayadi AA, Jones E, Churchman S, Baboolal T, Cuthbert R, El-Jawhari J, et al. Interleukin-22 drives the proliferation, migration and osteogenic differentiation of mesenchymal stem cells: a novel cytokine that could contribute 